\title{
BMJ Open Cefoxitin versus piperacillin- tazobactam as surgical antibiotic prophylaxis in patients undergoing pancreatoduodenectomy: protocol for a randomised controlled trial
}

To cite: Nevarez NM, Brajcich BC, Liu J, et al. Cefoxitin versus piperacillintazobactam as surgical antibiotic prophylaxis in patients undergoing pancreatoduodenectomy: protocol for a randomised controlled trial. BMJ Open 2021;11:e048398. doi:10.1136/ bmjopen-2020-048398

- Prepublication history for this paper is available online. To view these files, please visit the journal online (http://dx.doi org/10.1136/bmjopen-2020048398).

Received 27 December 2020 Revised 02 February 2021 Accepted 04 February 2021

D Check for updates

(c) Author(s) (or their employer(s)) 2021. Re-use permitted under CC BY-NC. No commercial re-use. See rights and permissions. Published by BMJ.

For numbered affiliations see end of article.

Correspondence to Dr Adam C Yopp; adam.yopp@utsouthwestern. edu

\section{ABSTRACT}

Introduction Although antibiotic prophylaxis is established in reducing postoperative surgical site infections (SSIs), the optimal antibiotic for prophylaxis in pancreatoduodenectomy (PD) remains unclear. The study objective is to evaluate if administration of piperacillintazobactam as antibiotic prophylaxis results in decreased 30-day SSI rate compared with cefoxitin in patients undergoing elective PD.

Methods and analysis This study will be a multiinstitution, double-arm, non-blinded randomised controlled superiority trial. Adults $\geq 18$ years consented to undergo PD for all indications who present to institutions participating in the National Surgical Quality Improvement Program Hepato-Pancreato-Biliary (NSQIP HPB) Collaborative will be included. Data collection will use the NSQIP HPB Collaborative Surgical Clinical Reviewers. Patients will be randomised to either $1-2 \mathrm{~g}$ intravenous cefoxitin or $3.375-4.5 \mathrm{~g}$ intravenous piperacillin-tazobactam within 60 min of surgical incision. The primary outcome will be 30-day postoperative SSI rate following PD. Secondary outcomes will include 30-day postoperative mortality; specific postoperative complication rate; and unplanned reoperation, length of stay, and hospital readmission. A subset of patients will have bacterial isolates and sensitivities of intraoperative bile cultures and SSIs. Postoperative SSIs and secondary outcomes will be analysed using logistic regression models with the primary predictor as the randomised treatment group. Additional adjustment will be made for preoperative biliary stent presence. Additionally, bacterial cultures and isolates will be summarised by presence of bacterial species and antibiotic sensitivities.

Ethics and dissemination This study is approved by the Institutional Review Board at Memorial Sloan Kettering Cancer Center. This trial will evaluate the effect of piperacillin-tazobactam compared with cefoxitin as antibiotic prophylaxis on the hazard of postoperative SSIs. The results will be disseminated regardless of the effect of the intervention on study outcomes. The manuscript describing the effect of the intervention will be submitted to a peer-reviewed journal when data collection and analyses are complete.
Strengths and limitations of this study

- A major strength of this study is the multiinstitutional, double-arm, randomised controlled trial design.

- A limitation of this study is that all perioperative care is at the discretion of the operating surgeon and is not standardised.

- All data will be collected through the American College of Surgeons National Surgical Quality Improvement Program, which is a strength for its ease of use but a limitation due to the variety of data included.

Trial registration number NCT03269994.

\section{INTRODUCTION}

Surgical site infections (SSIs) remain the most common and costly of all hospitalacquired infections (HAIs), accounting for nearly $20 \%$ of all HAIs. ${ }^{1}$ SSIs are associated with increased length of stay and a twofold to 11-fold increase in mortality. Despite rates of SSI in other complex abdominal operations decreasing, the SSI rate after pancreatoduodenectomy (PD) has remained unchanged, ranging from $11 \%$ to $48 \% .^{2}$ An analysis of data from the approximately 70 institutions within the Hepato-Pancreato-Biliary (HPB) Collaborative of the American College of Surgeons National Surgical Quality Improvement Program (ACS NSQIP) detailing 3592 PDs in 2015 revealed the rate of postoperative combined superficial incisional, deep incisional and organ/space SSIs to be $21.7 \%$ $(n=778)$. This SSI rate was nearly threefold the rate after colon resections, which was $8.9 \%$ in ACS NSQIP hospitals in 2015. ${ }^{3}$ Factors associated with the high rate of SSIs in patients undergoing PD include preoperative biliary 
stenting, malnutrition, receipt of neoadjuvant therapy and prolonged operative duration among others. ${ }^{4-7}$

In 2003, the Centers for Medicare and Medicaid Services initiated a programme to reduce preventable surgical complications including SSIs. ${ }^{8-10}$ This effort resulted in the creation of the Surgical Care Improvement Project (SCIP) measures. ${ }^{11-13}$ The three SCIP measures with regard to antibiotics and SSI prevention include (1) administration of antibiotics within 1 hour of incision time, (2) selection of appropriate antibiotic therapy and (3) discontinuation of antibiotics within 24 hours after surgery end time (or 48 hours for cardiac procedures). The current SCIP guidelines recommend a one-time dose of cefotetan, cefoxitin, ampicillin/sulbactam, or ertapenem or cefazolin or cefuroxime plus metronidazole with no evidence of B-lactam allergy as antimicrobial prophylaxis in PD; alternative agents in patients with a B-lactam allergy include clindamycin or vancomycin plus aminoglycoside or aztreonam or fluoroquinolone. ${ }^{13}$

Although administration of a first-generation or second-generation cephalosporin in patients undergoing PD remains the most common antibiotic regimen for surgical prophylaxis, observational studies have suggested that these regimens may provide inadequate coverage for Enterococcus spp, Escherichia coli, and Klebsiella spp. ${ }^{14-20}$ These organisms are the most common isolates from SSIs following PD, especially in patients what have had preoperative biliary stenting. ${ }^{14-20}$ Two retrospective studies compared the rate of SSI following PD when antimicrobial surgical prophylaxis was changed from the SCIP recommended cefoxitin (62\%), cefazolin and metronidazole $(15 \%)$ and clindamycin $(8 \%)$ to a piperacillin-tazobactam regimen. In both studies, the rate of SSI decreased twofold to fivefold, following use of piperacillin-tazobactam for antibiotic prophylaxis. ${ }^{21} 22$ Currently there is one prospective, non-randomised study using piperacillin-tazobactam for antibiotic prophylaxis; this study which showed that patients had a higher rate of HAI $(43 \%$ vs $33 \%$; $p=0.004)$, superficial SSI $(11 \%$ vs $2 \%$; $\mathrm{p}<0.001)$ and pneumonia $(16 \%$ vs $9 \% ; \mathrm{p}=0.006) .{ }^{23}$

\section{Objective}

The objective of this study is to determine if administration of piperacillin-tazobactam as antibiotic prophylaxis results in decreased rates of 30-day SSI as compared with the administration of cefoxitin (second-generation cephalosporin) in patients undergoing elective PD for all indications.

\section{METHODS AND ANALYSIS Overview}

This study will be a multicentre, double-arm randomised controlled superiority trial. The patients enrolled and consented will be randomised to receive either $1-2 \mathrm{~g}$ of intravenous cefoxitin or 3.375-4.5 g of intravenous piperacillin-tazobactam as surgical antibiotic prophylaxis for PD. We hypothesise that administration of piperacillin-tazobactam as antibiotic prophylaxis will reduce rates of SSI after PD compared with cefoxitin.

\section{Study setting}

This trial will be carried out among the 80 institutions in the USA participating in both the ACS NSQIP HPB Collaborative and ACS NSQIP Procedure Targeted Pancreatectomy Program. ACS NSQIP is a nationally validated, risk-adjusted, outcome-based effort to measure and improve surgical care. The programme employs a prospective data registry to quantify 30 -day, risk-adjusted surgical outcomes, which provide a standardised comparison of outcomes among all participating hospitals. ${ }^{24}$ In an internal analysis, 2210 PDs were conducted in 2015 specifically at these institutions participating in the ACS NSQIP Procedure Targeted Pancreatectomy Program. ${ }^{3}$

\section{Patient population and eligibility criteria}

The study population will consist of all patients $\geq 18$ years of age at the ACS NSQIP HPB Collaborative institutions undergoing elective PD for all indications (cancer and non-cancer). Exclusion criteria include patients undergoing a minimally invasive (laparoscopic or robotic) PD; known and documented allergies to any of the penicillins, cephalosporins or $\beta$-lactamase inhibitors; patients highly unlikely to undergo PD according to the surgeon's judgement, such as conditions amenable to pancreas enucleation or ampullectomy; patients with long-term glucocorticosteroid use; patients unable to provide informed consent; patients with renal insufficiency or failure with creatinine clearance $\leq 40 \mathrm{~mL} / \mathrm{min}$ or receiving haemodialysis or peritoneal dialysis; pregnant or nursing (lactating) women; patients with a known bacterial infection present at the time of surgery or who received antimicrobial therapy within 7 days prior to surgery.

\section{Recruitment}

This study will be open to all patients seen at the participating ACS NSQIP HPB Collaborative institutions who meet the eligibility criteria described above. Furthermore, to be a participating institution, the hospital must be part of the ACS NSQIP Procedure Targeted Pancreatectomy Program and agree to collect all PDs performed at their institution during the study period. The study was designed in conjunction with the Americas HepatoPancreato-Biliary Association Clinical Trials Committee and will be advertised within its confines for institutional participation. Patients will be identified from surgical clinics for treatment of their disease. After a discussion of the patient's disease and a formulation of the initial treatment plan, the physician-investigator or designee will describe the study to the patient. The protocol will be discussed in a private clinic room or office. Details including the risks and obligations of the subjects will be explained. For non-English speaking patients, an independent translator will be available to communicate the details of the protocol. Patients will be required to read, 
agree to and sign an Institutional Review Board-approved informed consent form prior to registration in this trial.

\section{Randomisation, allocation concealment and blinding}

After informed consent is obtained, site investigators at the HPB Collaborative institutions will contact the trial coordinating centre for randomisation. Patients will be randomised 1:1 to cefoxitin or piperacillin-tazobactam by the method of random permuted block, and patients will be stratified by presence of preoperative biliary stent. Randomisation will occur prior to the day of surgery to ensure institutional antibiotic availability. The assigned antibiotic will be administered within $60 \mathrm{~min}$ of incision for PD and discontinued within 24 hours of anaesthesia end time. Participants, surgical team, outcomes assessors, care providers (except anaesthesiologist) and biostatisticians will be blinded to study allocation status.

\section{Intervention}

After informed consent and randomisation, patients will receive one dose of either cefoxitin or piperacillin-tazobactam within $60 \mathrm{~min}$ of incision time, re-dosed every 2-4hours in the operating room until closure of the incision and discontinued within 24 hours after anaesthesia end time.

\section{Surgical conduct during and after PD}

Perioperative care will be at the discretion of the attending surgeon and will be provided to both cohorts. Care includes type of analgesia, type of incision, transfusion of blood components, use of drains, and deep venous thrombosis prophylaxis.

\section{Data collection}

Trained data abstractors, called surgical clinical reviewers, at each hospital participating in the ACS NSQIP collect patient information pertaining to demographics, operative details and postoperative outcomes from the clinical record using standardised definitions up to 30 days from the index operation. Outcomes are determined directly from the medical record, by communicating with any involved providers, or from the patient directly via mail or telephone. Operations are recorded using Common Procedural Terminology (CPT) codes. While outpatient operations are included, minor operations in freestanding surgery centres or in offices are not. Submitted data are periodically audited to ensure quality and reliability between abstractors. ${ }^{25}$

The data that will be collected on patient characteristics prior to PD include

Demographic data:

- Date of birth.

- Gender.

- Race.

- Hispanic ethnicity.

- Preferred language.

- Hospital admission date.

- Operation date.

- CPT code for operation.
- Inpatient/outpatient status.

- Origin status.

- Principal anaesthetic technique.

- Surgeon specialty.

- Surgeon National Provider Identification number.

- Height.

- Weight.

- Diabetes mellitus requiring therapy with non-insulin agents or insulin.

- Current smoker within 1 year of operation.

- Dyspnoea.

- Functional health status.

- Ventilator dependence.

- Chronic obstructive pulmonary disease.

- Ascites within 30 days prior to surgery.

- Congestive heart failure within 30 days prior to surgery.

- Hypertension requiring medication.

- Acute renal failure.

- Currently requiring or on dialysis.

- Disseminated cancer.

- Open wound (with or without infection).

- Steroid/immunosuppressant use for a chronic condition.

- $>10 \%$ loss of body weight in the 6 months prior to surgery.

- Bleeding disorders.

- Blood transfusions within 72 hours prior to surgery start time.

- Sepsis within 48 hours prior to surgery.

- Preoperative laboratory values (within 90 days of the operation): serum sodium, blood urea nitrogen, serum creatinine, albumin, total bilirubin, aspartate transaminase/serum glutamine-oxaloacetic transaminase, alkaline phosphatase, white blood cell count, haematocrit, platelets, international normalised ratio, partial thromboplastin time.

- American Society of Anesthesiology Classification.

- Presence of preoperative jaundice.

- Presence of a preoperative biliary stent.

- Receipt of chemotherapy within 90 days of the operation.

- Receipt of radiation therapy within 90 days of the operation.

Operative information:

- Emergency operation status.

- Wound classification.

- Surgical wound closure.

- Operation start and end times.

- Additional operations/procedures performed simultaneously on the same patient.

- Need for intraoperative blood transfusion within 72 hours of surgery start time.

- Operative approach.

- Type of surgical incision.

- Use of a wound protector.

- Pancreatic duct size at the time of the operation.

- Pancreatic gland texture at the time of the operation. 
- Type of pancreatic reconstruction performed, if any.

- Presence and type, if appropriate, of surgical drains.

- If surgical drains are placed, whether they are placed to suction.

- Need for vascular resection.

- Malignancy versus benign pathology.

- If malignant, pathologic American Joint Committee on Cancer stage.

- If benign, tumour size.

\section{Postoperative complications}

Postoperative complications will be defined according to the ACS NSQIP Standard Data Definitions and Pancreatectomy Data Definitions and will be collected up to 30 days from the index operation. Common postoperative complications that will be included are

- Superficial incisional SSI.

- Deep incisional SSI.

- Organ/space SSI.

- Wound disruption.

- Pneumonia.

- Intraoperative or postoperative unplanned intubation.

- Intraoperative or postoperative pulmonary embolism.

- On ventilator $>48$ hours.

- Progressive renal insufficiency.

- Acute renal failure requiring dialysis.

- Urinary tract infection.

- Intraoperative or postoperative stroke/cerebral vascular accident.

- Intraoperative or postoperative cardiac arrest requiring cardiopulmonary resuscitation (CPR).

- Intraoperative or postoperative myocardial infarction.

- Venous thrombosis requiring therapy.

- Sepsis.

- Clostridium difficile infection.

- Need for postoperative blood transfusion within 72 hours of surgery start time.

- Presence of pancreatic fistula.

- Presence of delayed gastric emptying.

- Unplanned reoperation.

- Acute hospital discharge date.

- Hospital discharge destination.

- Death during operation or postoperative death within 30 days of procedure.

- Date of death.

- Hospital readmission.

\section{Laboratory analyses}

Physician-investigators will be encouraged to collect bacterial cultures intraoperatively from the biliary system in patients undergoing PD at the time of biliary duct transection and from postoperative SSIs whenever feasible. These data will not be collected by the standard ACS NSQIP mechanism but will be studied in a retrospective fashion by participating institutions where surgeons routinely perform these cultures via chart review. Specifically, these correlative studies will be performed by institutions whose surgeons routinely culture bile and/or their postoperative SSIs.

\section{Study outcomes}

Primary outcome

- Rate of overall SSIs within the first 30 days after PD.

Secondary outcomes

- Rate of postoperative mortality within the first 30 days after PD.

- Rate of specific postoperative complications within the first 30 days after PD.

- Rates of hospital utilisation such as unplanned reoperation, hospital stay $>30$ days, and hospital readmission.

- Bacterial isolates and sensitivities of intraoperative bile cultures and SSIs.

\section{Statistical methods \\ Sample size calculation}

An internal analysis of data from the approximately 70 institutions within the HPB Collaborative of the ACS NSQIP detailing 3592 PDs in 2015 revealed the rate of postoperative combined superficial incisional, deep incisional and organ/space SSIs to be $21.7 \%(\mathrm{n}=778)$. Additional internal analysis of ACS NSQIP data that included additional hospitals revealed the overall SSI rate to be $20.4 \%$. The primary aim of this study is to determine if administration of piperacillin-tazobactam as surgical antibiotic prophylaxis results in decreased rates of SSI as compared with the administration of cefoxitin. To accomplish this aim, patients undergoing elective PD will be randomised to receive one of the two treatments and then will be followed for overall SSI occurrence for 30 days. The estimated reduction in SSI rate will be from 0.20 in the cefoxitin group to 0.13 in the piperacillin-tazobactam group. This study will use a two-sided type I error rate of 0.05 and a power of 0.8 . Two interim analyses will be planned when one-third and two-thirds of the patients have been accrued. The interim analysis is based on the O'Brien-Fleming spending function. One hundred and forty-eight patients per group will be accrued for a total of 297 patients in total. For the first interim analysis, 297 patients per group will be accrued for a total of 593 patients for the second interim analysis. At interim analysis, the null hypothesis of no difference between groups will be rejected if the $p$ value for the test was $<0.005$ and the trial would stop at that time. Otherwise, the trial will continue enrolling patients until 445 patients per group have been accrued, for a total of 890 patients overall. At the final analysis, the null hypothesis of no difference between groups will be rejected if the $p$ value for the test was $<0.048$.

The planned samples size of 890 is for resectable patients. Randomisation is done pre-surgery but it is expected that approximately $10 \%$ of patients who are taken to the operating room will be deemed unresectable. These patients will be ineligible and will be replaced. In 2015, 2210 PDs were conducted at institutions interested 
in participating, accounting for recruitment and eligibility considerations. Thus, we expect the study to be completed within 24 months. ${ }^{3}$

\section{Descriptive statistics}

Continuous variables will be described using means and SD for normally distributed data. For continuous variables with non-normally distributed data, medians and ranges will be used. Categorical data will be described using counts, proportions, and risk ratios with 95\% CIs.

\section{Planned outcome analysis}

\section{Primary outcome}

All analyses will be conducted among resected patients. For the primary endpoint, a logistic regression model will be fitted where the outcome is SSI yes or no, the primary predictor is the randomised treatment group and additional adjustment is made for presence of preoperative biliary stent.

\section{Secondary outcomes}

All secondary endpoints are binary and will be analysed using logistic regression models with the secondary endpoint as the outcome, randomisation group as the primary predictor, and adjustment for the presence of a biliary stent preoperatively. Bacterial cultures and isolates from certain participating institutions will be summarised. Specifically, these data include whether bacterial cultures were positive and, if positive, which bacterial species are present and their antibiotic sensitivities.

\section{Patient and public involvement}

Patients or the public were not involved in the design of our research and will not be involved in conduct, reporting or dissemination of our research.

\section{DISCUSSION}

SSI is one of the most common postoperative hospitalacquired complications following PD with significant healthcare costs. Previous studies have shown bacterial cultures from SSI after PD are more likely to be sensitive to piperacillin-tazobactam. ${ }^{21}{ }^{22}$ This study will attempt to demonstrate piperacillin-tazobactam as superior for antibiotic prophylaxis compared with cefoxitin for patients undergoing PD. This study also will use the NSQIP registry platform for data collection, a novel approach for the conduct of a randomised clinical trial and the blueprint for future planned trials using this platform.

\section{ETHICS AND DISSEMINATION}

\section{Research ethics approval}

This study has received approval from the Institutional Review Board at Memorial Sloan Kettering Cancer Center. The study protocol, informed consent, form and other submitted documents were reviewed and approved.

\section{Confidentiality}

Patient data will be managed by the ACS NSQIP. The ACS NSQIP is a Health Insurance Portability and Accountability Act-compliant registry whose data is managed by Outcome Sciences LLC, a QuintilesIMS company. All patient data are identifiable at each hospital. However, data submitted to the ACS NSQIP for quality improvement purposes, and for the purposes of this study, are de-identified.

\section{Dissemination policy}

The results of this study will be disseminated regardless of the effect of the intervention on study outcomes. The manuscript describing the effect of the intervention will be submitted to a peer-reviewed journal when data collection and analyses are complete.

\section{Author affiliations}

${ }^{1}$ Department of Surgery, The University of Texas Southwestern Medical Center, Dallas, Texas, USA

${ }^{2}$ American College of Surgeons, Chicago, Illinois, USA

${ }^{3}$ Department of Surgery, University of Chicago Medical Center, Chicago, Illinois, USA ${ }^{4}$ Department of Surgery, Rutgers Cancer Institute of New Jersey, New Brunswick, New Jersey, USA

${ }^{5}$ Department of Surgery, Memorial Sloan Kettering Cancer Center, New York, New York, USA

\section{Twitter Nicole M Nevarez @nicnev91}

Contributors AY and MID coordinated and actively participated in all the phases of trial design, statistical analysis plan and drafting of the protocol including this manuscript. NMN wrote this manuscript. HAP, BCB, JL, RE, CYK critically revised the design, methodology, statistical analysis plan and this manuscript. All authors agreed with submission of the final version of this manuscript for publication.

Funding This study will be funded in part through a research grant from the Americas Hepato-Pancreato-Biliary Association.

Competing interests None declared.

Patient and public involvement Patients and/or the public were not involved in the design, or conduct, or reporting, or dissemination plans of this research.

Patient consent for publication Not required.

Provenance and peer review Not commissioned; externally peer reviewed.

Open access This is an open access article distributed in accordance with the Creative Commons Attribution Non Commercial (CC BY-NC 4.0) license, which permits others to distribute, remix, adapt, build upon this work non-commercially, and license their derivative works on different terms, provided the original work is properly cited, appropriate credit is given, any changes made indicated, and the use is non-commercial. See: http://creativecommons.org/licenses/by-nc/4.0/.

\section{ORCID iD}

Nicole M Nevarez http://orcid.org/0000-0002-1076-3402

\section{REFERENCES}

1 Magill SS, Edwards JR, Bamberg W, et al. Multistate pointprevalence survey of health care-associated infections. $N$ Engl J Med 2014;370:1198-208.

2 Smit LC, Bruins MJ, Patijn GA, et al. Infectious complications after major abdominal cancer surgery: in search of improvable risk factors. Surg Infect 2016;17:683-93.

3 American College of Surgeons National Surgical Quality Improvement Program Hepato-Pancreato-Biliary (ACS NSQIP HPB). Procedure targeted pancreatectomy program; 2018.

4 Suragul W, Rungsakulkij N, Vassanasiri W, et al. Predictors of surgical site infection after pancreaticoduodenectomy. BMC Gastroenterol 2020;20:1-10. 
5 Ceppa EP, Pitt HA, House MG. Reducing surgical site infections in hepatopancreatobiliary surgery 2013;15:384-91.

6 Povoski SP, Karpeh MS, Conlon KC, et al. Preoperative biliary drainage: impact on intraoperative bile cultures and infectious morbidity and mortality after pancreaticoduodenectomy. $J$ Gastrointest Surg 1999;3:496-505.

7 Howard TJ, Yu J, Greene RB, et al. Influence of bactibilia after preoperative biliary stenting on postoperative infectious complications. J Gastrointest Surg 2006;10:523-31.

8 Cataife G, Weinberg DA, Wong H-H, et al. The effect of surgical care improvement project (SCIP) compliance on surgical site infections (SSI). Med Care 2014;52:S66-73.

9 Hawn MT, Vick CC, Richman J, et al. Surgical site infection prevention: time to move beyond the surgical care improvement program. Ann Surg 2011;254:494-9.

10 Stulberg JJet al. Adherence to surgical care improvement project measures and the association with postoperative infections. JAMA 2010;303:2479-85.

11 Anderson DJ, Podgorny K, Berríos-Torres SI, et al. Strategies to prevent surgical site infections in acute care hospitals: 2014 update. Infect Control Hosp Epidemiol 2014;35:605-27.

12 Mangram AJ, Horan TC, Pearson ML, et al. Guideline for prevention of surgical site infection, 1999. Infect Control Hosp Epidemiol 1999;20:247-80

13 Bratzler DW, Dellinger EP, Olsen KM, et al. Clinical practice guidelines for antimicrobial prophylaxis in surgery. Am J Health Syst Pharm 2013;70:195-283.

14 Sudo T, Murakami Y, Uemura K, et al. Perioperative antibiotics covering bile contamination prevent abdominal infectious complications after pancreatoduodenectomy in patients with preoperative biliary drainage. World J Surg 2014;38:2952-9.

15 Barreto SG, Singh MK, Sharma S, et al. Determinants of surgical site infections following pancreatoduodenectomy. World J Surg 2015;39:2557-63.

16 Cortes A, Sauvanet A, Bert F, et al. Effect of bile contamination on immediate outcomes after pancreaticoduodenectomy for tumor. $J$ Am Coll Surg 2006;202:93-9.
17 Sourrouille I, Gaujoux S, Lacave G, et al. Five days of postoperative antimicrobial therapy decreases infectious complications following pancreaticoduodenectomy in patients at risk for bile contamination. HPB 2013;15:473-80.

18 Gavazzi F, Ridolfi C, Capretti G, et al. Role of preoperative biliary stents, bile contamination and antibiotic prophylaxis in surgical site infections after pancreaticoduodenectomy. BMC Gastroenterol 2016;16:1-11.

19 Limongelli P, Pai M, Bansi D, et al. Correlation between preoperative biliary drainage, bile duct contamination, and postoperative outcomes for pancreatic surgery. Surgery 2007;142:313-8.

20 Fong ZV, McMillan MT, Marchegiani G, et al. Discordance between perioperative antibiotic prophylaxis and wound infection cultures in patients undergoing pancreaticoduodenectomy. JAMA Surg 2016;151:432.

21 Donald GW, Sunjaya D, Lu X, et al. Perioperative antibiotics for surgical site infection in pancreaticoduodenectomy: does the SCIP-approved regimen provide adequate coverage? Surgery 2013;154:190-6.

22 Kondo K, Chijiiwa K, Ohuchida J, et al. Selection of prophylactic antibiotics according to the microorganisms isolated from surgical site infections (SSIs) in a previous series of surgeries reduces SSI incidence after pancreaticoduodenectomy. $J$ Hepatobiliary Pancreat Sci 2013;20:286-93.

23 De Pastena M, Paiella S, Azzini AM, et al. Antibiotic prophylaxis with piperacillin-tazobactam reduces post-operative infectious complication after pancreatic surgery: an interventional, NonRandomized study. Surg Infect 2020. doi:10.1089/sur.2020.260. [Epub ahead of print: 23 Oct 2020].

24 Parikh $\mathrm{P}$, Shiloach M, Cohen ME, et al. Pancreatectomy risk calculator: an ACS-NSQIP resource. HPB 2010;12:488-97.

25 Shiloach M, Frencher SK, Steeger JE, et al. Toward robust information: data quality and inter-rater reliability in the American College of surgeons national surgical quality improvement program. $J$ Am Coll Surg 2010;210:6-16. 